Article

\title{
Robust Approximate Optimality Conditions for Uncertain Nonsmooth Optimization with Infinite Number of Constraints
}

\author{
Xiangkai Sun ${ }^{1} \mathbb{O}$, Hongyong $\mathrm{Fu}^{2, *}$ and Jing Zeng ${ }^{1}$ \\ 1 Chongqing Key Laboratory of Social Economy and Applied Statistics, College of Mathematics and Statistics, \\ Chongqing Technology and Business University, Chongqing 400067, China; sunxk@ctbu.edu.cn (X.S.); \\ yiyuexue219@163.com (J.Z.) \\ 2 China Research Institute of Enterprise Governed by Law, Southwest University of Political Science and Law, \\ 301 Baosheng Street, Chongqing 401120, China \\ * Correspondence: fuhongyong@swupl.edu.cn; Tel.: +86-023-6725-8383
}

Received: 3 December 2018; Accepted: 21 December 2018; Published: 23 December 2018

check for updates

\begin{abstract}
This paper deals with robust quasi approximate optimal solutions for a nonsmooth semi-infinite optimization problems with uncertainty data. By virtue of the epigraphs of the conjugates of the constraint functions, we first introduce a robust type closed convex constraint qualification. Then, by using the robust type closed convex constraint qualification and robust optimization technique, we obtain some necessary and sufficient optimality conditions for robust quasi approximate optimal solution and exact optimal solution of this nonsmooth uncertain semi-infinite optimization problem. Moreover, the obtained results in this paper are applied to a nonsmooth uncertain optimization problem with cone constraints.
\end{abstract}

Keywords: approximate quasi-solutions; optimality conditions; robust nonsmooth semi-infinite optimization

\section{Introduction}

Let $T$ be a nonempty infinite index set, $C \subseteq \mathbb{R}^{n}$ be a nonempty convex set, and let $f, g_{t}: \mathbb{R}^{n} \rightarrow \mathbb{R}$, $t \in T$, be continuous functions. We focus on the following semi-infinite optimization problem with infinite number of inequality constraints

$$
\text { SIP }\left\{\begin{array}{l}
\min f(x) \\
\text { s.t. } g_{t}(x) \leq 0, t \in T, \\
x \in C .
\end{array}\right.
$$

The feasible set of SIP is defined by

$$
\overline{\mathcal{F}}:=\left\{x \in C \mid g_{t}(x) \leq 0, \text { for all } t \in T\right\} .
$$

It is well known that this modeling of problems as SIP is an very interesting research topic in mathematical programming due to the wide range of its applications in various fields such as Chebyshev approximation, engineering design, and optimal control, etc. For the last decades, many papers have been devoted to investigate SIP from different points of view. We refer the readers to the references [1-11] for more details. However, most practical optimization problems are often 
contaminated with uncertainty data. Thus, it is meaning to study the theory and application of SIP with uncertainty data. In this paper, we consider the uncertainty case of SIP

$$
\text { USIP }\left\{\begin{array}{c}
\min f(x, u) \\
\text { s.t. } g_{t}\left(x, v_{t}\right) \leq 0, t \in T, \\
x \in C,
\end{array}\right.
$$

where $f: \mathbb{R}^{n} \times \mathbb{R}^{m} \rightarrow \mathbb{R}$, and $g_{t}: \mathbb{R}^{n} \times \mathbb{R}^{q} \rightarrow \mathbb{R}, t \in T$, are continuous functions. The uncertain parameters $u$ and $v_{t}, t \in T$, belong to the convex compact sets $\mathcal{U} \subseteq \mathbb{R}^{m}$ and $\mathcal{V}_{t} \subseteq \mathbb{R}^{q}, t \in T$, respectively.

Nowadays, robust optimization technique has been recognized as one of the powerful deterministic methodology that investigates an optimization problem with data uncertainty in the objective or constraint functions. Following this methodology, many interesting results have been obtained for different kinds of uncertain optimization problems, see, for example, [12-25]. Here, we only mention the works on optimality due to $[17,19,21,23,25]$. More precisely, by virtue of a so-called Extended Nonsmooth Mangasarian-Fromovitz constraint qualification, Lee and Pham [17] obtained a necessary optimality condition for a class of nonsmooth optimization problems in the face of uncertainty data. Chuong [19] obtained some necessary and sufficient optimality conditions for robust Pareto efficient solutions of an uncertain multiobjective optimization problem in terms of multipliers and limiting subdifferential properties. By using a robust type constraint qualifications, Sun et al. [21] investigated some characterizations of robust optimal solutions of an fractional optimization problem under uncertainty data both in the objective and constraint functions. Fakhar et al. [23] deduced some optimality conditions for robust (weakly) efficient solutions of a class of uncertain nonsmooth multiobjective optimization problems in terms of a new concept of generalized convexity. They also presented the viability of their methodology for portfolio optimization. Wei et al. [25] established some weak alternative theorems and optimality conditions for a general scalar robust optimization problem by using the image space analysis approach. However, there is a few papers to deal with robust approximate optimal solutions of uncertain optimization problems, see, for example, [26-29]. Lee and Lee $[26,29]$ established optimality theorems and duality results of robust approximate optimal solutions for uncertain convex optimization problems under closed convex cone constraint qualifications (see also [27]). Sun et al. [28] introduced a robust type closed convex constraint qualification condition and then established some optimality conditions for robust approximate optimal solutions of a convex optimization problem with uncertainty data.

Motivated by the work reported in [27-29], this paper is devoted to deal with robust quasi approximate optimal solution of USIP by virtue of its robust counterpart

$$
\operatorname{RUSIP}\left\{\begin{array}{l}
\min \left\{\max _{u \in \mathcal{U}} f(x, u)\right\} \\
\text { s.t. } g_{t}\left(x, v_{t}\right) \leq 0, \forall\left(t, v_{t}\right) \in \operatorname{gph} \mathcal{V}, \\
x \in C,
\end{array}\right.
$$

where $\mathcal{V}: T \rightarrow 2^{\mathbb{R} q}$ is an uncertainty set-valued mapping with $\mathcal{V}(t):=\mathcal{V}_{t}$, for all $t \in T$. We make two major contributions to invistigate robust quasi approximate optimal solutions for USIP. By using the epigraphs of the conjugate functions, we first introduce some new robust type constraint qualification conditions. Then, we obtain some robust forms of necessary and sufficient optimality conditions for quasi approximate optimal solutions of USIP, which gives a new generalization of the approximate optimality conditions for semi-infinite optimization problems to uncertain semi-infinite optimization problems. Moreover, we apply the approach used in this paper to investigate uncertain optimization problems with cone constraints. We also show that several results on characterizations of (robust) approximate optimal solution of (uncertain) optimization problems reported in recent literature can be obtained by the use of our approach. 
We organize this paper as follows. Section 2 give some basic definitions and preliminary results used in this paper. Sections 3 and 4 obtain necessary and sufficient optimality conditions for robust quasi approximate optimal solutions of USIP. Section 5 is devoted to apply the proposed methods to an uncertain optimization problem with cone constraints.

\section{Preliminaries}

In this paper, we recall some notations and preliminary results, see, e.g., $[3,30]$. Let $\mathbb{R}^{n}$ be the Euclidean space of dimension $n$ and let $\mathbb{R}_{+}^{n}$ be the nonnegative orthant of $\mathbb{R}^{n}$. The symbol $\mathbb{B}^{*}$ stands for the closed unit ball of $\mathbb{R}^{n}$. The inner product in $\mathbb{R}^{n}$ is defined by $\langle\cdot, \cdot\rangle$. The norm of an element $\xi$ of $\mathbb{R}^{n}$ is given by

$$
\|\xi\|:=\sup \left\{\langle\xi, d\rangle \mid d \in \mathbb{R}^{n},\|d\| \leq 1\right\} .
$$

Let $D \subseteq \mathbb{R}^{n}$. The closure (resp. convex hull, convex cone hull) of $D$ is denoted by cl $D$ (resp. co $D$, cone $D$ ). The dual cone of $D$ is defined by

$$
D^{*}=\left\{x^{*} \in \mathbb{R}^{n} \mid\left\langle x^{*}, x\right\rangle \geq 0, \forall x \in D\right\} .
$$

The indicator function $\delta_{D}: \mathbb{R}^{n} \rightarrow \mathbb{R} \cup\{+\infty\}$ of $D$ is defined by

$$
\delta_{D}(x)=\left\{\begin{array}{l}
0, \quad \text { if } x \in D \\
+\infty, \text { if } x \notin D
\end{array}\right.
$$

For the nonempty infinite index set $T$. Let $\mathbb{R}^{(T)}$ be the following linear space [1],

$$
\mathbb{R}^{(T)}:=\left\{\lambda=\left(\lambda_{t}\right)_{t \in T} \mid \lambda_{t}=0 \text { for all } t \in T \text { except for finitely many } \lambda_{t} \neq 0\right\} .
$$

The positive cone of $\mathbb{R}^{(T)}$ is defined by

$$
\mathbb{R}_{+}^{(T)}:=\left\{\lambda \in \mathbb{R}^{(T)} \mid \lambda_{t} \geq 0 \text { for all } t \in T\right\} .
$$

The supporting set of $\lambda \in \mathbb{R}^{(T)}$ is defined by

$$
T(\lambda):=\left\{t \in T \mid \lambda_{t} \neq 0\right\},
$$

which is a finite subset of $T$.

For an extended real-valued function $f: \mathbb{R}^{n} \rightarrow \mathbb{R} \cup\{+\infty\} . \quad f$ is said to be proper, if $\operatorname{dom} f=\left\{x \in \mathbb{R}^{n} \mid f(x)<+\infty\right\} \neq \varnothing$. The epigraph of $f$ is defined by epi $f=\left\{(x, r) \in \mathbb{R}^{n} \times\right.$ $\mathbb{R} \mid f(x) \leq r\} . f$ is said to be a convex function if epi $f$ is a convex set. The function $f$ is said to be concave whenever $-f$ is convex. More generally, $f$ is said to be lower semicontinuous if epi $f$ is closed. The Legendre-Fenchel conjugate function of $f$ is $f^{*}: \mathbb{R}^{n} \rightarrow \mathbb{R} \cup\{ \pm \infty\}$ defined by

$$
f^{*}\left(x^{*}\right)=\sup _{x \in \mathbb{R}^{n}}\left\{\left\langle x^{*}, x\right\rangle-f(x)\right\}, \forall x^{*} \in \mathbb{R}^{n}
$$

For any $\varepsilon \geq 0$, the $\varepsilon$-subdifferential of $f$ at $\bar{x} \in \operatorname{dom} f$ is given by

$$
\partial^{\varepsilon} f(\bar{x})=\left\{x^{*} \in \mathbb{R}^{n} \mid f(x)-f(\bar{x}) \geq\left\langle x^{*}, x-\bar{x}\right\rangle-\varepsilon, \forall x \in \mathbb{R}^{n}\right\} .
$$

If $\varepsilon=0$, the set $\partial f(\bar{x}):=\partial^{0} f(\bar{x})$ is the subdifferential of $f$ at $\bar{x} \in \operatorname{dom} f$, that is,

$$
\partial f(\bar{x})=\left\{x^{*} \in \mathbb{R}^{n} \mid f(x)-f(\bar{x}) \geq\left\langle x^{*}, x-\bar{x}\right\rangle, \forall x \in \mathbb{R}^{n}\right\} .
$$


Lemma 1 ([31]). Let $f: \mathbb{R}^{n} \rightarrow \mathbb{R} \cup\{+\infty\}$ be a proper, lower semicontinuous, and convex function, and let $\bar{x} \in \operatorname{dom} f$. Then,

$$
\text { epi } f^{*}=\bigcup_{\varepsilon \geq 0}\left\{(\xi,\langle\xi, \bar{x}\rangle+\varepsilon-f(\bar{x})) \mid \xi \in \partial^{\varepsilon} f(\bar{x})\right\} \text {. }
$$

Lemma 2 ([3]). Let $I$ be an arbitrary index set and let $f_{i}: \mathbb{R}^{n} \rightarrow \mathbb{R} \cup\{+\infty\}, i \in I$, be proper, lower semicontinuous and convex functions on $\mathbb{R}^{n}$. Assume that there exists $x_{0} \in \mathbb{R}^{n}$, such that $\sup _{i \in I} f_{i}\left(x_{0}\right)<\infty$. Then,

$$
\operatorname{epi}\left(\sup _{i \in I} f_{i}\right)^{*}=\mathrm{cl}\left(\operatorname{co} \bigcup_{i \in I} \operatorname{epi} f_{i}^{*}\right)
$$

where $\sup _{i \in I} f_{i}: \mathbb{R}^{n} \rightarrow \mathbb{R} \cup\{+\infty\}$ is defined by $\left(\sup _{i \in I} f_{i}\right)(x)=\sup _{i \in I} f_{i}(x)$ for all $x \in \mathbb{R}^{n}$.

Lemma 3 ([3]). Let $f_{1}, f_{2}: \mathbb{R}^{n} \rightarrow \mathbb{R} \cup\{+\infty\}$ be proper, and convex functions such that dom $f_{1} \cap$ dom $f_{2} \neq \varnothing$.

(i) If $f_{1}$ and $f_{2}$ are lower semicontinuous, then,

$$
\text { epi }\left(f_{1}+f_{2}\right)^{*}=\mathrm{cl}\left(\text { epi } f_{1}^{*}+\text { epi } f_{2}^{*}\right) \text {. }
$$

(ii) If one of $f_{1}$ and $f_{2}$ is continuous at some $\bar{x} \in \operatorname{dom} f_{1} \cap \operatorname{dom} f_{2}$, then,

$$
\text { epi }\left(f_{1}+f_{2}\right)^{*}=\text { epi } f_{1}^{*}+\text { epi } f_{2}^{*} \text {. }
$$

\section{Necessary Approximate Optimality Conditions}

This section is devoted to establish some necessary optimality conditions for a robust quasi approximate optimal solution of USIP. The following conceptions and results will be used in this sequel.

Definition 1. The robust feasible set of USIP is given by

$$
\mathcal{F}:=\left\{x \in C \mid g_{t}\left(x, v_{t}\right) \leq 0, \forall v_{t} \in \mathcal{V}_{t}, t \in T\right\}
$$

Definition 2. (i) Let $\varepsilon \geq 0$. A point $\bar{x} \in \mathcal{F}$ is called a robust quasi $\varepsilon$-optimal solution of USIP, iff $\bar{x} \in \mathcal{F}$ is a quasi $\varepsilon$-optimal solution of RUSIP, i.e.,

$$
\max _{u \in \mathcal{U}} f(\bar{x}, u) \leq \max _{u \in \mathcal{U}} f(x, u)+\sqrt{\varepsilon}\|x-\bar{x}\|, \forall x \in \mathcal{F}
$$

(ii) A point $\bar{x} \in \mathcal{F}$ is called a robust optimal solution of USIP, iff $\bar{x} \in \mathcal{F}$ is an optimal solution of RUSIP, i.e.,

$$
\max _{u \in \mathcal{U}} f(\bar{x}, u) \leq \max _{u \in \mathcal{U}} f(x, u), \forall x \in \mathcal{F}
$$

Remark 1. If $\mathcal{U}$ is a singleton set, the robust quasi $\varepsilon$-optimal solution coincides the concept defined by Lee and Lee [29]. Moreover, if $\mathcal{U}$ and $\mathcal{V}_{t}, t \in T$ are singletons, the robust quasi $\varepsilon$-optimal solution of USIP deduces to be the usual one of quasi $\varepsilon$-optimal solution of SIP, that is

$$
f(\bar{x}) \leq f(x)+\sqrt{\varepsilon}\|x-\bar{x}\|, \forall x \in \overline{\mathcal{F}} .
$$

For more details, please see [5,32].

The following constraint qualification will be used in the study of robust quasi approximate optimal solution of USIP. 
Definition 3. We say that robust type constraint qualification condition RCQC holds, iff

$$
\bigcup_{v \in \mathcal{V}, \lambda \in \mathbb{R}_{+}^{(T)}} \text { epi }\left(\sum_{t \in T} \lambda_{t} g_{t}\left(\cdot, v_{t}\right)\right)^{*}+\text { epi } \delta_{C}^{*} \text { is a closed convex set, }
$$

where $v \in \mathcal{V}$ means that $v$ is a selection of $\mathcal{V}$, i.e., $v: T \rightarrow \mathbb{R}^{q}$ and $v_{t} \in \mathcal{V}_{t}$ for all $t \in T$.

Remark 2. In the special case when $C=\mathbb{R}^{n}, \mathrm{RCQC}$ coincides the so-called closed convex cone constraint qualification defined by Lee and Lee [29].

Now, following [29] , we give some characterizations of RCQC.

Proposition 1. Let $g_{t}: \mathbb{R}^{n} \times \mathbb{R}^{q} \rightarrow \mathbb{R}, t \in T$, be continuous functions. Assume that $\mathcal{V}_{t} \subseteq \mathbb{R}^{n}, t \in T$, is convex, and for any $v_{t} \in \mathcal{V}_{t}, g_{t}\left(\cdot, v_{t}\right)$ is a convex function, and for any $x \in \mathbb{R}^{n}, g_{t}(x, \cdot)$ is concave on $\mathcal{V}_{t}$. Then,

$$
\bigcup_{v \in \mathcal{V}, \lambda \in \mathbb{R}_{+}^{(T)}} \operatorname{epi}\left(\sum_{t \in T} \lambda_{t} g_{t}\left(\cdot, v_{t}\right)\right)^{*}+\operatorname{epi} \delta_{C}^{*}
$$

is a convex set.

Proof. By ([29] Proposition 2.5) and ([28] Proposition 3.6), we can easily get the desired result.

Naturally, we can obtain the following result by virtue of ([29] Proposition 2.6).

Proposition 2. Let $T$ be a compact metric space, and let $\mathcal{V}$ be compact-valued and uniformly upper semi-continuous on $T$. Let $g_{t}: \mathbb{R}^{n} \times \mathbb{R}^{q} \rightarrow \mathbb{R}, t \in T$, be continuous functions such that for each $v_{t} \in \mathcal{V}_{t}, g_{t}\left(\cdot, v_{t}\right)$ is a convex function. Suppose that there exists $\hat{x} \in \mathbb{R}^{n}$ such that $g_{t}\left(\hat{x}, v_{t}\right)<0$, for any $v_{t} \in \mathcal{V}_{t}, t \in T$. Then,

$$
\bigcup_{v \in \mathcal{V}, \lambda \in \mathbb{R}_{+}^{(T)}} \operatorname{epi}\left(\sum_{t \in T} \lambda_{t} g_{t}\left(\cdot, v_{t}\right)\right)^{*}+\operatorname{epi} \delta_{C}^{*}
$$

is a closed set.

Now, we present a robust Farkas Lemma for convex functions. Since its proof is similar to ([29] Lemma 3.1), we omit it.

Lemma 4. Let $h: \mathbb{R}^{n} \rightarrow \mathbb{R}$ be a convex function, and let $g_{t}: \mathbb{R}^{n} \times \mathbb{R}^{q} \rightarrow \mathbb{R}, t \in T$, be continuous functions such that for any $v_{t} \in \mathcal{V}_{t}, g_{t}\left(\cdot, v_{t}\right)$ is a convex function. Then, the following statements are equivalent:

(i) $\quad\left\{x \in C \mid g_{t}\left(x, v_{t}\right) \leq 0, \forall v_{t} \in \mathcal{V}_{t}, t \in T\right\} \subseteq\left\{x \in \mathbb{R}^{n} \mid h(x) \geq 0\right\}$.

(ii) $\quad(0,0) \in$ epi $h^{*}+\operatorname{clco}\left(\bigcup_{v \in \mathcal{V}, \lambda \in \mathbb{R}_{+}^{(T)}}\right.$ epi $\left(\sum_{t \in T} \lambda_{t} g_{t}\left(\cdot, v_{t}\right)\right)^{*}+$ epi $\left.\delta_{C}^{*}\right)$.

Now, we gives a necessary approximate optimality condition for a robust quasi $\varepsilon$-optimal solution of USIP under the condition RCQC.

Theorem 1. Let $\varepsilon \geq 0$. Let $f: \mathbb{R}^{n} \times \mathbb{R}^{m} \rightarrow \mathbb{R}$ be a continuous convex-concave function, and let $g_{t}:$ $\mathbb{R}^{n} \times \mathbb{R}^{q} \rightarrow \mathbb{R}, t \in T$, be continuous functions such that $g_{t}\left(\cdot, v_{t}\right)$ is convex on $\mathbb{R}^{n}$, for any $v_{t} \in \mathcal{V}_{t}$. 
Assume that RCQC holds. If $\bar{x} \in \mathcal{F}$ is a robust quasi E-optimal solution of USIP, then, there exist $\bar{u} \in \mathcal{U}$, $\left(\bar{\lambda}_{t}\right)_{t \in T} \in \mathbb{R}_{+}^{(T)}$, and $\bar{v}_{t} \in \mathcal{V}_{t}, t \in T$ such that

$$
0 \in \partial_{x} f(\bar{x}, \bar{u})+\sum_{t \in T} \partial_{x}\left(\bar{\lambda}_{t} g_{t}\right)\left(\bar{x}, \bar{v}_{t}\right)+\partial \delta_{C}(\bar{x})+\sqrt{\varepsilon} \mathbb{B}^{*},
$$

and

$$
g_{t}\left(\bar{x}, \bar{v}_{t}\right)=0, \forall t \in T(\bar{\lambda}) .
$$

Proof. Assume that $\bar{x} \in \mathcal{F}$ is a robust quasi $\varepsilon$-optimal solution of USIP. Then,

$$
\max _{u \in \mathcal{U}} f(\bar{x}, u) \leq \max _{u \in \mathcal{U}} f(x, u)+\sqrt{\varepsilon}\|x-\bar{x}\|, \quad \forall x \in \mathcal{F} .
$$

This means that

$$
\mathcal{F} \subseteq\left\{x \in C \mid \max _{u \in \mathcal{U}} f(x, u)+\sqrt{\varepsilon}\|x-\bar{x}\|-\max _{u \in \mathcal{U}} f(\bar{x}, u) \geq 0\right\}
$$

Let

$$
\varphi(x):=\max _{u \in \mathcal{U}} f(x, u)+\sqrt{\varepsilon}\|x-\bar{x}\|-\max _{u \in \mathcal{U}} f(\bar{x}, u)
$$

By Lemma 4, we get

$$
(0,0) \in \operatorname{epi} \varphi^{*}+\operatorname{clco}\left(\bigcup_{v \in \mathcal{V}, \lambda \in \mathbb{R}_{+}^{(T)}} \operatorname{epi}\left(\sum_{t \in T} \lambda_{t} g_{t}\left(\cdot, v_{t}\right)\right)^{*}+\text { epi } \delta_{C}^{*}\right) .
$$

Since RCQC holds, it follows from (1) and Lemma 3 that

$$
(0,0) \in \operatorname{epi} \varphi^{*}+\bigcup_{v \in \mathcal{V}, \lambda \in \mathbb{R}_{+}^{(T)}} \sum_{t \in T} \operatorname{epi}\left(\lambda_{t} g_{t}\left(\cdot, v_{t}\right)\right)^{*}+\operatorname{epi} \delta_{C}^{*} .
$$

On the other hand, by using Lemma 2 and the similar method of ([27] Theorem 1),

$$
\text { epi } \varphi^{*}=\operatorname{cl}\left(\operatorname{co~} \bigcup_{u \in \mathcal{U}} \operatorname{epi}(f(\cdot, u))^{*}\right)+\sqrt{\varepsilon} \mathbb{B}^{*} \times\left[\sqrt{\varepsilon}\|\bar{x}\|+\max _{u \in \mathcal{U}} f(\bar{x}, u),+\infty\right)
$$

Moreover, since $f$ is a continuous convex-concave function, it is easy to see $\bigcup_{u \in \mathcal{U}}$ epi $(f(\cdot, u))^{*}$ is a closed convex set. Then,

$$
\text { epi } \varphi^{*}=\bigcup_{u \in \mathcal{U}} \text { epi }(f(\cdot, u))^{*}+\sqrt{\varepsilon} \mathbb{B}^{*} \times\left[\sqrt{\varepsilon}\|\bar{x}\|+\max _{u \in \mathcal{U}} f(\bar{x}, u),+\infty\right)
$$

Together with (2) and (3), we have

$$
\begin{aligned}
&(0,-\sqrt{\varepsilon}\|\bar{x}\|\left.-\max _{u \in \mathcal{U}} f(\bar{x}, u)\right) \in \bigcup_{u \in \mathcal{U}} \text { epi }(f(\cdot, u))^{*}+ \\
& \bigcup_{v \in \mathcal{V}, \lambda \in \mathbb{R}_{+}^{(T)}} \sum_{t \in T} \text { epi }\left(\lambda_{t} g_{t}\left(\cdot, v_{t}\right)\right)^{*}+\text { epi } \delta_{C}^{*}+\sqrt{\varepsilon} \mathbb{B}^{*} \times \mathbb{R}_{+} .
\end{aligned}
$$


Therefore, there exist $\bar{u} \in \mathcal{U},\left(\bar{\lambda}_{t}\right)_{t \in T} \in \mathbb{R}_{+}^{(T)}$, and $\bar{v}_{t} \in \mathcal{V}_{t}, t \in T$ such that

$$
\left(0,-\sqrt{\varepsilon}\|\bar{x}\|-\max _{u \in \mathcal{U}} f(\bar{x}, u)\right) \in \operatorname{epi}(f(\cdot, \bar{u}))^{*}+\sum_{t \in T} \operatorname{epi}\left(\bar{\lambda}_{t} g_{t}\left(\cdot, \bar{v}_{t}\right)\right)^{*}+\operatorname{epi} \delta_{C}^{*}+\sqrt{\varepsilon} \mathbb{B}^{*} \times \mathbb{R}_{+} .
$$

This follows that there exist $\left(\xi_{0}^{*}, r_{0}\right) \in$ epi $(f(\cdot, \bar{u}))^{*},\left(\xi_{t}^{*}, r_{t}\right) \in$ epi $\left(\bar{\lambda}_{t} g_{t}\left(\cdot, \bar{v}_{t}\right)\right)^{*},\left(\gamma^{*}, s\right) \in$ epi $\delta_{C}^{*}$, and $\left(b^{*}, b\right) \in \mathbb{B}^{*} \times \mathbb{R}_{+}$, such that

$$
\left(0,-\sqrt{\varepsilon}\|\bar{x}\|-\max _{u \in \mathcal{U}} f(\bar{x}, u)\right)=\left(\xi_{0}^{*}+\sum_{t \in T} \xi_{t}^{*}+\gamma^{*}+\sqrt{\varepsilon} b^{*}, r_{0}+\sum_{t \in T} r_{t}+s+b\right) .
$$

Moreover, by Lemma 1 , there exist $\varepsilon_{0} \geq 0, \varepsilon_{t} \geq 0, t \in T$, and $\varepsilon_{s} \geq 0$ such that

$$
\begin{gathered}
\xi_{0}^{*} \in \partial_{x}^{\varepsilon_{0}} f(\bar{x}, \bar{u}) \text {, and } r_{0}=\left\langle\xi_{0}^{*}, \bar{x}\right\rangle+\varepsilon_{0}-f(\bar{x}, \bar{u}), \\
\xi_{t}^{*} \in \partial_{x}^{\varepsilon_{t}}\left(\bar{\lambda}_{t} g_{t}\right)\left(\bar{x}, \bar{v}_{t}\right), \text { and } r_{t}=\left\langle\xi_{t}^{*}, \bar{x}\right\rangle+\varepsilon_{t}-\left(\bar{\lambda}_{t} g_{t}\right)\left(\bar{x}, \bar{v}_{t}\right), \\
\gamma^{*} \in \partial^{\varepsilon_{s}} \delta_{C}(\bar{x}) \text {, and } s=\left\langle\gamma^{*}, \bar{x}\right\rangle+\varepsilon_{s} .
\end{gathered}
$$

From (4), we deduce that

$$
0 \in \partial_{x}^{\varepsilon_{0}} f(\bar{x}, \bar{u})+\sum_{t \in T} \partial_{x}^{\varepsilon_{t}}\left(\bar{\lambda}_{t} g_{t}\right)\left(\bar{x}, \bar{v}_{t}\right)+\partial^{\varepsilon_{s}} \delta_{C}(\bar{x})+\sqrt{\varepsilon} \mathbb{B}^{*}
$$

and

$$
\begin{aligned}
& -\sqrt{\varepsilon}\|\bar{x}\|-\max _{u \in \mathcal{U}} f(\bar{x}, u) \\
= & r_{0}+\sum_{t \in T} r_{t}+s+b \\
= & \left\langle\xi_{0}^{*}+\sum_{t \in T} \xi_{t}^{*}+\gamma^{*}, \bar{x}\right\rangle+\varepsilon_{0}+\sum_{t \in T} \varepsilon_{t}+\varepsilon_{s}-f(\bar{x}, \bar{u})-\sum_{t \in T} \bar{\lambda}_{t} g_{t}\left(\bar{x}, \bar{v}_{t}\right)+b \\
= & -\sqrt{\varepsilon}\left\langle b^{*}, \bar{x}\right\rangle+\varepsilon_{0}+\sum_{t \in T} \varepsilon_{t}+\varepsilon_{s}-f(\bar{x}, \bar{u})-\sum_{t \in T} \bar{\lambda}_{t} g_{t}\left(\bar{x}, \bar{v}_{t}\right)+b \\
\geq & -\sqrt{\varepsilon}\left\langle b^{*}, \bar{x}\right\rangle+\varepsilon_{0}+\sum_{t \in T} \varepsilon_{t}+\varepsilon_{s}-\max _{u \in \mathcal{U}} f(\bar{x}, u)-\sum_{t \in T} \bar{\lambda}_{t} g_{t}\left(\bar{x}, \bar{v}_{t}\right)+b .
\end{aligned}
$$

Together with $\bar{\lambda}_{t} g_{t}\left(\bar{x}_{,} \bar{v}_{t}\right) \leq 0$, we get

$$
0 \leq \varepsilon_{0}+\sum_{t \in T} \varepsilon_{t}+\varepsilon_{s}-\sum_{t \in T} \bar{\lambda}_{t} g_{t}\left(\bar{x}, \bar{v}_{t}\right) \leq \sqrt{\varepsilon}\left\langle b^{*}, \bar{x}\right\rangle-\sqrt{\varepsilon}\|\bar{x}\|-b \leq 0 .
$$

Then, $\varepsilon_{0}=\varepsilon_{t}=\varepsilon_{s}=0$ and $\bar{\lambda}_{t} g_{t}\left(\bar{x}, \bar{v}_{t}\right)=0$. Thus, it follows from (5) that

$$
0 \in \partial_{x} f(\bar{x}, \bar{u})+\sum_{t \in T} \partial_{x}\left(\bar{\lambda}_{t} g_{t}\right)\left(\bar{x}, \bar{v}_{t}\right)+\partial \delta_{C}(\bar{x})+\sqrt{\varepsilon} \mathbb{B}^{*}
$$

This completes the proof.

Remark 3. It is worth observing that robust approximate optimality conditions for USIP obtained in Theorem 1 seems to be new (the same as for Theorems 2, 3 and 4). The robust approximate optimality result similar to the one in Theorems 1 and 2 appeared in ([27] Theorem 3.2) when $C=\mathbb{R}^{n}, \mathcal{U}$ is singleton and $T$ is a nonempty finite index set.

Now, we present an numerical example to illustrate our necessary robust approximate optimality condition for USIP. 
Example 1 ([29]). Let $\mathbb{R}^{n}=\mathbb{R}^{m}=\mathbb{R}^{q}=\mathbb{R}, C=\mathbb{R}_{+}, \mathcal{U}=[0,1]$, and $\mathcal{V}_{t}=\left[1-\frac{t}{2}, 1+\frac{t}{2}\right]$ for any $t \in T:=[0,1]$. Moreover, for any $x \in \mathbb{R}, u \in \mathcal{U}$ and $v_{t} \in \mathcal{V}_{t}$, let

$$
f(x, u)=x^{2}-u x
$$

and

$$
g_{t}\left(x, v_{t}\right)=t x^{2}-v_{t} x .
$$

Then, for USIP, it is easy to verify that $\mathcal{F}=\left[0, \frac{1}{2}\right]$, and RCQC holds. So, the conditions of Theorem 1 are satisfied.

On the other hand, let $\varepsilon=2$ and $\bar{x}=0 \in \mathcal{F}$. Obviously, $\bar{x}$ is a robust quasi $\varepsilon$-optimal solution of USIP. Moreover, for instance, there exist $\bar{u}=0, \bar{\lambda}_{t}=1, t=0, \bar{\lambda}_{t}=0, t \in(0,1]$, and $\bar{v}_{t}=1+\frac{t}{4}, t \in[0,1]$ such that

$$
0 \in \partial_{x} f(\bar{x}, \bar{u})+\sum_{t \in T} \partial_{x}\left(\bar{\lambda}_{t} g_{t}\right)\left(\bar{x}, \bar{v}_{t}\right)+\partial \delta_{C}(\bar{x})+\sqrt{\varepsilon} \mathbb{B}^{*},
$$

and

$$
g_{t}\left(\bar{x}, \bar{v}_{t}\right)=0, \forall t \in T(\bar{\lambda}) .
$$

Corollary 1. Let $T$ be a compact metric space, and let $\mathcal{V}$ be compact-valued and uniformly upper semi-continuous on $T$. Let $f: \mathbb{R}^{n} \times \mathbb{R}^{m} \rightarrow \mathbb{R}$ be a continuous convex-concave function, and let $g_{t}: \mathbb{R}^{n} \times \mathbb{R}^{q} \rightarrow \mathbb{R}, t \in T$, be continuous functions such that for each $v_{t} \in \mathcal{V}_{t}, g_{t}\left(\cdot, v_{t}\right)$ is a convex function, and for each $x \in \mathbb{R}^{n}, g_{t}(x, \cdot)$ is concave on $\mathcal{V}_{t}$. Suppose that there exists $\hat{x} \in \mathbb{R}^{n}$ such that $g_{t}\left(\hat{x}, v_{t}\right)<0$, for any $v_{t} \in \mathcal{V}_{t}, t \in T$. If $\bar{x} \in \mathcal{F}$ is a robust quasi $\varepsilon$-optimal solution of USIP, then, there exist $\bar{u} \in \mathcal{U},\left(\bar{\lambda}_{t}\right)_{t \in T} \in \mathbb{R}_{+}^{(T)}$, and $\bar{v}_{t} \in \mathcal{V}_{t}, t \in T$ such that

$$
0 \in \partial_{x} f(\bar{x}, \bar{u})+\sum_{t \in T} \partial_{x}\left(\bar{\lambda}_{t} g_{t}\right)\left(\bar{x}, \bar{v}_{t}\right)+\partial \delta_{C}(\bar{x})+\sqrt{\varepsilon} \mathbb{B}^{*},
$$

and

$$
g_{t}\left(\bar{x}, \bar{v}_{t}\right)=0, \forall t \in T(\bar{\lambda}) .
$$

Proof. Combing Proposition 1, Proposition 2 and Theorem 1, we can easily obtain the desired result.

When $\mathcal{U}$ and $\mathcal{V}$ are singletons, the following result holds naturally.

Corollary 2. Let $\varepsilon \geq 0$. Let $f: \mathbb{R}^{n} \rightarrow \mathbb{R}$ be a convex function, and let $g_{t}: \mathbb{R}^{n} \rightarrow \mathbb{R}, t \in T$, be continuous convex functions. Assume that cone $\left(\bigcup_{t \in T}\right.$ epi $\left.g_{t}^{*}\right)+$ epi $\delta_{C}^{*}$ is a closed set. If $\bar{x} \in \overline{\mathcal{F}}$ is a quasi e-optimal solution of SIP, then, there exist $\left(\bar{\lambda}_{t}\right)_{t \in T} \in \mathbb{R}_{+}^{(T)}$, such that

$$
0 \in \partial_{x} f(\bar{x})+\sum_{t \in T} \bar{\lambda}_{t} \partial_{x} g_{t}(\bar{x})+\partial \delta_{C}(\bar{x})+\sqrt{\varepsilon} \mathbb{B}^{*}, \text { and } g_{t}(\bar{x})=0, \forall t \in T(\bar{\lambda}) .
$$

The following theorem establish necessary condition for robust optimal solution of USIP. This result can be considered as a version of robust optimality condition for nonsmooth and nonlinear semi-infinite optimization problems which have not yet been considered in the literature.

Theorem 2. Let $f: \mathbb{R}^{n} \times \mathbb{R}^{m} \rightarrow \mathbb{R}$ be a continuous convex-concave function, and let $g_{t}: \mathbb{R}^{n} \times \mathbb{R}^{q} \rightarrow \mathbb{R}$, $t \in T$, be continuous functions such that $g_{t}\left(\cdot, v_{t}\right)$ is convex on $\mathbb{R}^{n}$, for any $v_{t} \in \mathcal{V}_{t}$. Assume that RCQC holds. 
If $\bar{x} \in \mathcal{F}$ is a robust optimal solution of USIP, then, there exist $\bar{u} \in \mathcal{U},\left(\bar{\lambda}_{t}\right)_{t \in T} \in \mathbb{R}_{+}^{(T)}$, and $\bar{v}_{t} \in \mathcal{V}_{t}, t \in T$ such that

$$
0 \in \partial_{x} f(\bar{x}, \bar{u})+\sum_{t \in T} \partial_{x}\left(\bar{\lambda}_{t} g_{t}\right)\left(\bar{x}, \bar{v}_{t}\right)+\partial \delta_{C}(\bar{x})
$$

and

$$
g_{t}\left(\bar{x}, \bar{v}_{t}\right)=0, \forall t \in T(\bar{\lambda})
$$

The particular case of Theorem 1 corresponding to the cases where $\varepsilon=0, \mathcal{U}$ and $\mathcal{V}$ are singletons are of special interest. The interested reader is referred to $[4,6-8,10]$ for necessary optimality conditions of SIP in terms of different conditions.

Corollary 3. Let $f: \mathbb{R}^{n} \rightarrow \mathbb{R}$ be a convex function, and let $g_{t}: \mathbb{R}^{n} \rightarrow \mathbb{R}, t \in T$, be continuous convex functions. Assume that cone $\left(\bigcup_{t \in T}\right.$ epi $\left.g_{t}^{*}\right)+$ epi $\delta_{C}^{*}$ is a closed set. If $\bar{x} \in \overline{\mathcal{F}}$ is a optimal solution of SIP, then, there exist $\left(\bar{\lambda}_{t}\right)_{t \in T} \in \mathbb{R}_{+}^{(T)}$, such that

$$
0 \in \partial_{x} f(\bar{x})+\sum_{t \in T} \bar{\lambda}_{t} \partial_{x} g_{t}(\bar{x})+\partial \delta_{C}(\bar{x}), \text { and } \quad g_{t}(\bar{x})=0, \forall t \in T(\bar{\lambda}) .
$$

\section{Sufficient Approximate Optimality Conditions}

This section is devoted to give some sufficient approximate optimality condition for a robust quasi approximate optimal solution of USIP. Now, we first introduce a new concept of generalized robust approximate KKT conditions for USIP.

Definition 4. Let $\varepsilon \geq 0$. A point $(\bar{x}, \bar{\lambda}, \bar{u}, \bar{v}) \in \mathcal{F} \times \mathbb{R}_{+}^{(T)} \times \mathcal{U} \times \mathcal{V}$ is said to satisfy the generalized robust approximate KKT conditions for USIP, iff

$$
0 \in \partial_{x} f(\bar{x}, \bar{u})+\sum_{t \in T} \partial_{x}\left(\bar{\lambda}_{t} g_{t}\right)\left(\bar{x}, \bar{v}_{t}\right)+\partial \delta_{C}(\bar{x})+\sqrt{\varepsilon} \mathbb{B}^{*},
$$

and

$$
g_{t}\left(\bar{x}, \bar{v}_{t}\right)=0, t \in T(\bar{\lambda}) .
$$

Remark 4. Let $\varepsilon=0$ in Definition 4. Then, the generalized robust approximate KKT conditions for USIP coincides with the generalized robust KKT conditions for USIP.

Theorem 3. Let $\varepsilon \geq 0$. Suppose that $(\bar{x}, \bar{\lambda}, \bar{u}, \bar{v}) \in \mathcal{F} \times \mathbb{R}_{+}^{(T)} \times \mathcal{U} \times \mathcal{V}$ satisfies the generalized robust approximate KKT conditions and $f(\bar{x}, \bar{u})=\max _{u \in \mathcal{U}} f_{i}(\bar{x}, u)$. Then, $\bar{x} \in \mathcal{F}$ is a robust quasi E-optimal solution of USIP.

Proof. Since $(\bar{x}, \bar{\lambda}, \bar{u}, \bar{v}) \in \mathcal{F} \times \mathbb{R}_{+}^{(T)} \times \mathcal{U} \times \mathcal{V}$ satisfies the generalized robust approximate KKT conditions, there exist $\xi_{0}^{*} \in \partial_{x} f(\bar{x}, \bar{u}), \xi_{t}^{*} \in \partial_{x}\left(\bar{\lambda}_{t} g_{t}\right)\left(\bar{x}, \bar{v}_{t}\right)$, and $\gamma^{*} \in \partial \delta_{C}(\bar{x})$ and $\zeta^{*} \in \mathbb{B}^{*}$, such that

$$
\xi_{0}^{*}+\sum_{t \in T} \xi_{t}^{*}+\gamma^{*}+\sqrt{\varepsilon} \zeta^{*}=0
$$

Since $\xi_{0}^{*} \in \partial_{x} f(\bar{x}, \bar{u}), \xi_{t}^{*} \in \partial_{x}\left(\bar{\lambda}_{t} g_{t}\right)\left(\bar{x}, \bar{v}_{t}\right)$, and $\gamma^{*} \in \partial \delta_{C}(\bar{x})$, we obtain that, for any $x \in \mathcal{F}$,

$$
\begin{gathered}
f(x, \bar{u})-f(\bar{x}, \bar{u}) \geq\left\langle\xi_{0}^{*}, x-\bar{x}\right\rangle, \\
\left(\bar{\lambda}_{t} g_{t}\right)\left(x, \bar{v}_{t}\right)-\left(\bar{\lambda}_{t} g_{t}\right)\left(\bar{x}, \bar{v}_{t}\right) \geq\left\langle\xi_{t}^{*}, x-\bar{x}\right\rangle,
\end{gathered}
$$


and

$$
\delta_{C}(x)-\delta_{C}(\bar{x}) \geq\left\langle\gamma^{*}, x-\bar{x}\right\rangle
$$

Then,

$$
\begin{aligned}
& f(x, \bar{u})-f(\bar{x}, \bar{u})+\sum_{t \in T} \bar{\lambda}_{t} g_{t}\left(x, \bar{v}_{t}\right)-\sum_{t \in T} \bar{\lambda}_{t} g_{t}\left(\bar{x}, \bar{v}_{t}\right) \\
\geq & \left\langle\xi_{0}^{*}+\sum_{t \in T} \xi_{t}^{*}+\gamma^{*}, x-\bar{x}\right\rangle, \forall x \in \mathcal{F} .
\end{aligned}
$$

Moreover, together with $\bar{\lambda}_{t} g_{t}\left(x, \bar{v}_{t}\right) \leq 0$ and (8), we get,

$$
f(x, \bar{u})-f(\bar{x}, \bar{u})-\sum_{t \in T} \bar{\lambda}_{t} g_{t}\left(\bar{x}, \bar{v}_{t}\right) \geq-\sqrt{\varepsilon}\left\langle\zeta^{*}, x-\bar{x}\right\rangle, \forall x \in \mathcal{F} .
$$

It follows from $(7), f(\bar{x}, \bar{u})=\max _{u \in \mathcal{U}} f_{i}(\bar{x}, u)$ and $f(x, \bar{u}) \leq \max _{u \in \mathcal{U}} f_{i}(x, u)$ that

$$
\max _{u \in \mathcal{U}} f_{i}(x, u)-\max _{u \in \mathcal{U}} f_{i}(\bar{x}, u) \geq-\sqrt{\varepsilon}\left\langle\zeta^{*}, x-\bar{x}\right\rangle, \forall x \in \mathcal{F} .
$$

Note that $\left\langle\zeta^{*}, x-\bar{x}\right\rangle \leq\|x-\bar{x}\|, \forall x \in \mathcal{F}$. Thus,

$$
\max _{u \in \mathcal{U}} f_{i}(x, u)-\max _{u \in \mathcal{U}} f_{i}(\bar{x}, u) \geq-\sqrt{\varepsilon}\|x-\bar{x}\|, \forall x \in \mathcal{F},
$$

Thus, $\bar{x}$ is a robust $\varepsilon$-optimal solution of USIP and the proof is complete.

Remark 5. It is worth observing that if, in addition, $\mathcal{U}$ is singleton and $T$ is a nonempty finite index set, the approximate optimality conditions given in Theorem 3 was established in [27]. So, our results can be regarded as a generalization of the results obtained in [27].

In the case that $\mathcal{U}$ and $\mathcal{V}$ are singletons, we get the following optimality conditions which have been studied in [5] under different kinds of constraint qualifications.

Corollary 4. Consider the problem $\mathrm{SIP}$, and let $\varepsilon \geq 0$ and $\bar{x} \in \overline{\mathcal{F}}$. Let $f: \mathbb{R}^{n} \rightarrow \mathbb{R}$ be convex at $\bar{x}$, and let $g_{t}: \mathbb{R}^{n} \rightarrow \mathbb{R}, t \in T$, be continuous convex functions. If there exists $\left(\bar{\lambda}_{t}\right)_{t \in T} \in \mathbb{R}_{+}^{(T)}$ such that

$$
0 \in \partial f(\bar{x})+\sum_{t \in T} \bar{\lambda}_{t} \partial g_{t}(\bar{x})+\partial \delta_{C}(\bar{x})+\sqrt{\varepsilon} \mathbb{B}^{*} \text {, and } g_{t}(\bar{x})=0, t \in T(\bar{\lambda}) .
$$

Then, $\bar{x} \in \overline{\mathcal{F}}$ is a quasi $\varepsilon$-optimal solution of SIP.

The following theorem establish a sufficient condition for robust optimal solution of USIP.

Theorem 4. Let $\varepsilon \geq 0$. Suppose that $(\bar{x}, \bar{\lambda}, \bar{u}, \bar{v}) \in \mathcal{F} \times \mathbb{R}_{+}^{(T)} \times \mathcal{U} \times \mathcal{V}$ satisfies the generalized robust KKT conditions and $f(\bar{x}, \bar{u})=\max _{u \in \mathcal{U}} f_{i}(\bar{x}, u)$. Then, $\bar{x} \in \mathcal{F}$ is a robust optimal solution of USIP.

Similarly, when $\varepsilon=0, \mathcal{U}$ and $\mathcal{V}$ are singletons, the following sufficient optimality conditions for SIP are obtained. Please see $[4,6-8,10]$ for more details.

Corollary 5. Consider the problem $\mathrm{SIP}$, and $\bar{x} \in \overline{\mathcal{F}}$. Let $f: \mathbb{R}^{n} \rightarrow \mathbb{R}$ be convex at $\bar{x}$, and let $g_{t}: \mathbb{R}^{n} \rightarrow \mathbb{R}$, $t \in T$, be continuous convex functions. If there exists $\left(\bar{\lambda}_{t}\right)_{t \in T} \in \mathbb{R}_{+}^{(T)}$ such that

$$
0 \in \partial f(\bar{x})+\sum_{t \in T} \bar{\lambda}_{t} \partial g_{t}(\bar{x})+\partial \delta_{C}(\bar{x}), \text { and } g_{t}(\bar{x})=0, t \in T(\bar{\lambda}) .
$$


Then, $\bar{x} \in \overline{\mathcal{F}}$ is a optimal solution of SIP.

\section{Applications}

In this section, we apply the obtained results to an uncertain optimization problem with cone constraints. Let $C \subseteq \mathbb{R}^{n}$ be a nonempty convex set, and let $K \subseteq \mathbb{R}^{p}$ be a nonempty closed and convex cone. Consider an uncertain conic optimization problem

$$
\text { UCOP }\left\{\begin{array}{l}
\min f(x, u) \\
\text { s.t. } g(x, v) \in-K, \\
x \in C,
\end{array}\right.
$$

where $f: \mathbb{R}^{n} \times \mathbb{R}^{m} \rightarrow \mathbb{R}$ and $g: \mathbb{R}^{n} \times \mathbb{R}^{q} \rightarrow \mathbb{R}^{p}$ are continuous functions. The uncertain parameters $u$ and $v$ belong to the convex and compact uncertain sets $\mathcal{U} \subseteq \mathbb{R}^{m}$ and $\mathcal{V} \subseteq \mathbb{R}^{q}$, respectively.

As in [3], for each $\lambda \in K^{*},\langle\lambda, g\rangle$ will be denoted by $(\lambda g)$. Note that for any $x \in C$ and $v \in \mathcal{V}$, $g(x, v) \in-K$ if and only if $(\lambda g)(x, v) \leq 0, \forall \lambda \in K^{*}$. Then, UCOP can be reformulated as an example of USIP by setting

$$
T:=K^{*}, \quad g_{\lambda}\left(x, v_{\lambda}\right):=(\lambda g)(x, v), \quad \text { for any } \lambda \in T=K^{*} .
$$

In this section, we also use $\mathcal{F}$ to denote the feasible set of UCOP:

$$
\mathcal{F}:=\left\{x \in C \mid(\lambda g)(x, v) \leq 0, \forall v \in \mathcal{V}, \lambda \in K^{*}\right\}=\{x \in C \mid g(x, v) \in-K, \forall v \in \mathcal{V}\} .
$$

Moreover, let $\beta:=\left(\beta_{\lambda}\right)_{\lambda \in K^{*}} \in \mathbb{R}_{+}^{\left(K^{*}\right)}$, for any $\lambda \in K^{*}$. Then,

$$
\widetilde{\lambda}:=\sum_{\lambda \in K^{*}} \beta_{\lambda} \lambda \in K^{*},
$$

and so

$$
\bigcup_{v \in \mathcal{V}, \beta \in \mathbb{R}_{+}^{\left(K^{*}\right)}} \operatorname{epi}\left(\sum_{\lambda \in K^{*}} \beta_{\lambda}(\lambda g)(\cdot, v)\right)^{*}=\bigcup_{v \in \mathcal{V}, \tilde{\lambda} \in K^{*}} \operatorname{epi}((\widetilde{\lambda} g)(\cdot, v))^{*} .
$$

Thus, RCQC deduces to the constraint qualification RCCCQ introduced in [28], that is

$$
\bigcup_{v \in \mathcal{V}, \lambda \in K^{*}} \text { epi }((\lambda g)(\cdot, v))^{*}+\text { epi } \delta_{C}^{*} \text { is closed and convex. }
$$

Similarly, we can get the corresponding results for robust quasi $\varepsilon$-optimal solutions of UCOP.

Theorem 5. Let $\varepsilon \geq 0$. Let $f: \mathbb{R}^{n} \times \mathbb{R}^{m} \rightarrow \mathbb{R}$ be a continuous convex-concave function, and let $g$ : $\mathbb{R}^{n} \times \mathbb{R}^{q} \rightarrow \mathbb{R}^{p}$, be a continuous function such that $g(\cdot, v)$ is $K$-convex on $\mathbb{R}^{n}$, for any $v \in \mathcal{V}$. Assume that RCCCQ holds. If $\bar{x} \in \mathcal{F}$ is a robust quasi $\varepsilon$-optimal solution of $\mathrm{UCOP}$, then, there exist $\bar{u} \in \mathcal{U}, \bar{v} \in \mathcal{V}$, and $\bar{\lambda} \in K^{*}$, such that

$$
0 \in \partial_{x} f(\bar{x}, \bar{u})+\partial_{x}(\bar{\lambda} g)(\bar{x}, \bar{v})+\partial \delta_{C}(\bar{x})+\sqrt{\varepsilon} \mathbb{B}^{*},
$$

and

$$
\bar{\lambda} g(\bar{x}, \bar{v})=0
$$

The following theorem establish necessary condition for robust optimal solution of UCOP. 
Theorem 6. Let $f: \mathbb{R}^{n} \times \mathbb{R}^{m} \rightarrow \mathbb{R}$ be a continuous convex-concave function, and let $g: \mathbb{R}^{n} \times \mathbb{R}^{q} \rightarrow \mathbb{R}^{p}$ be a continuous function such that $g(\cdot, v)$ is $K$-convex on $\mathbb{R}^{n}$, for any $v \in \mathcal{V}$. Assume that RCCCQ holds. If $\bar{x} \in \mathcal{F}$ is a robust optimal solution of $\mathrm{UCOP}$, then, there exist $\bar{u} \in \mathcal{U}, \bar{v} \in \mathcal{V}$, and $\bar{\lambda} \in K^{*}$, such that

$$
0 \in \partial_{x} f(\bar{x}, \bar{u})+\partial_{x}(\bar{\lambda} g)(\bar{x}, \bar{v})+\partial \delta_{C}(\bar{x})
$$

and

$$
\bar{\lambda} g(\bar{x}, \bar{v})=0
$$

Definition 5. Let $\varepsilon \geq 0$. A point $(\bar{x}, \bar{\lambda}, \bar{u}, \bar{v}) \in \mathcal{F} \times K^{*} \times \mathcal{U} \times \mathcal{V}$ satisfies the generalized robust approximate KKT conditions for UCOP, iff

$$
0 \in \partial_{x} f(\bar{x}, \bar{u})+\partial_{x}(\bar{\lambda} g)(\bar{x}, \bar{v})+\partial \delta_{C}(\bar{x})+\sqrt{\varepsilon} \mathbb{B}^{*},
$$

and

$$
\bar{\lambda} g(\bar{x}, \bar{v})=0
$$

Remark 6. Note that if $\varepsilon=0$ in Definition 5, we obtain the concept of generalized robust KKT conditions for UCOP.

Similarly, we obtain the following sufficient optimality conditions for UCOP.

Theorem 7. Let $\varepsilon \geq 0$. Suppose that $(\bar{x}, \bar{\lambda}, \bar{u}, \bar{v}) \in \mathcal{F} \times K^{*} \times \mathcal{U} \times \mathcal{V}$ satisfies the generalized robust approximate $K K T$ conditions and $f(\bar{x}, \bar{u})=\max _{u \in \mathcal{U}} f_{i}(\bar{x}, u)$. Then, $\bar{x} \in \mathcal{F}$ is a robust quasi E-optimal solution of UCOP.

Theorem 8. Suppose that $(\bar{x}, \bar{\lambda}, \bar{u}, \bar{v}) \in \mathcal{F} \times K^{*} \times \mathcal{U} \times \mathcal{V}$ satisfies the generalized robust KKT conditions and $f(\bar{x}, \bar{u})=\max _{u \in \mathcal{U}} f_{i}(\bar{x}, u)$. Then, $\bar{x} \in \mathcal{F}$ is a robust optimal solution of UCOP.

Remark 7. In the case that $\mathcal{U}$ is a singleton, optimality conditions of other kinds of robust approximate optimal solutions for UCOP has been considered in [28].

\section{Conclusions}

In this paper, a nonsmooth semi-infinite optimization problem under data uncertainty USIP is considered. By using a new robust type constraint qualification condition RCQC and the notions of the subdifferential of convex functions, some necessary and sufficient approximate optimality conditions for robust quasi $\varepsilon$-optimal solutions of USIP are established. The results obtained in this paper improve the corresponding results reported in recent literature.

Our research paves the way for further study. It would be interesting to consider other concepts of approximate optimal solutions, such as almost robust (quasi) approximate optimal solution, or almost robust regular approximate optimal solution, for USIP in the future. On the other hand, since fractional semi-infinite optimization is one of an important model for semi-infinite optimization problems, so it could be possible to investigate fractional semi-infinite optimization problems with data uncertainty in the future.

Author Contributions: Conceptualization, X.S.; Writing-original draft preparation, X.S.; Writing-review and editing, X.S., H.F., and J.Z.; Funding acquisition, X.S., and J.Z.

Funding: This research was supported by the Basic and Advanced Research Project of Chongqing (cstc2017jcyjBX0032, cstc2016jcyjA0219), the National Natural Science Foundation of China (11701057), the Science 
and Technology Research Program of Chongqing Municipal Education Commission (KJQN201800837), and the Program for University Innovation Team of Chongqing (CXTDX201601026).

Conflicts of Interest: The authors declare no conflict of interest.

\section{References}

1. Goberna, M.; López, M. Linear Semi-Infinite Optimization; Wiley: Chichester, UK, 1998.

2. Reemtsen, R.; Ruckmann, J.J. Semi-Infinite Programming; Kluwer: Boston, MA, USA, 1998.

3. Boț, R.I. Conjugate Duality in Convex Optimization; Springer: Berlin, Germany, 2010.

4. Fang, D.H.; Li, C.; Ng, K.F. Constraint qualifications for extended Farkas's lemmas and Lagrangian dualities in convex infinite programming. SIAM J. Optim. 2009, 20, 1311-1332. [CrossRef]

5. Son, T.Q.; Strodiot, J.J.; Nguyen, V.H. $\varepsilon$-Optimality and $\varepsilon$-Lagrangian duality for a nonconvex programming problem with an infinite number of constraints. J. Optim. Theory Appl. 2009, 141, 389-409. [CrossRef]

6. Fang, D.H.; Li, C.; Ng, K.F. Constraint qualifications for optimality conditions and total Lagrange dualities in convex infinite programming. Nonlinear Anal. 2010, 73, 1143-1159. [CrossRef]

7. Kim, D.S.; Son, T.Q. Characterizations of solutions sets of a class of nonconvex semi-infinite programming problems. J. Nonlinear Convex Anal. 2011, 12, 429-440.

8. Sun, X.K. Regularity conditions characterizing Fenchel-Lagrange duality and Farkas-type results in DC infinite programming. J. Math. Anal. Appl. 2014, 414, 590-611. [CrossRef]

9. Long, X.J.; Peng, Z.Y.; Wang, X.F. Characterizations of the solution set for nonconvex semi-infinite programming problems. J. Nonlinear Convex Anal. 2016, 17, 251-265.

10. Long, X.J.; Xiao, Y.B.; Huang, N.J. Optimality conditions of approximate solutions for nonsmooth semi-infinite programming problems. J. Oper. Res. Soc. China 2018, 6, 289-299. [CrossRef]

11. Kim, D.S.; Son, T.Q. An approach to $\epsilon$-duality theorems for nonconvex semi-infinite multiobjective optimization problems. Taiwan J. Math. 2018, 22, 1261-1287. [CrossRef]

12. Ben-Tal, A.; Nemirovski, A. Robust optimization-methodology and applications. Math. Program. Ser. B 2002, 92, 453-480. [CrossRef]

13. Ben-Tal, A.; Ghaoui, L.E.; Nemirovski, A. Robust Optimization; Princeton Series in Applied Mathematics; Princeton University Press: Princeton, NJ, USA, 2009.

14. Jeyakumar, V.; Li, G.Y. Strong duality in robust convex programming: complete characterizations. SIAM J. Optim. 2010, 20, 3384-3407. [CrossRef]

15. Bertsimas, D.; Brown, D.B.; Caramanis, C. Theory and applications of robust optimization. SIAM Rev. 2011, 53, 464-501. [CrossRef]

16. Bọt, R.I.; Jeyakumar, V.; Li, G.Y. Robust duality in parametric convex optimization. Set Valued Var. Anal. 2013, 21, 177-189. [CrossRef]

17. Lee, G.M.; Pham, T.S. On nonsmooth optimality theorems for robust optimization problems. Bull. Korean Math. Soc. 2014, 51, 287-301. [CrossRef]

18. Jeyakumar, V.; Lee, G.M.; Li, G.Y. Characterizing robust solution sets of convex programs under data uncertainty. J. Optim. Theory Appl. 2015, 164, 407-435. [CrossRef]

19. Chuong, T.D. Optimality and duality for robust multiobjective optimization problems. Nonlinear Anal. 2016, 134, 127-143. [CrossRef]

20. Dinh, N.; Goberna, M.A.; López, M.A.; Volle, M. A unified approach to robust convex infinite optimization duality. J. Optim. Theory Appl. 2017, 174, 650-685. [CrossRef]

21. Sun, X.K.; Long, X.J.; Fu, H.Y.; Li, X.B. Some characterizations of robust optimal solutions for uncertain fractional optimization and applications. J. Ind. Manag. Optim. 2017, 13, 803-824. [CrossRef]

22. Dinh, N.; Long, D.H. Complete characterizations of robust strong duality for robust vector optimization problems. Vietnam J. Math. 2018, 46, 293-328. [CrossRef]

23. Fakhar, M.; Mahyarinia, M.R.; Zafarani, J. On nonsmooth robust multiobjective optimization under generalized convexity with applications to portfolio optimization. Eur. J. Oper. Res. 2018, 265, 39-48. [CrossRef]

24. Li, X.B.; Wang, S. Characterizations of robust solution set of convex programs with uncertain data. Optim. Lett. 2018, 12, 1387-1402. [CrossRef] 
25. Wei, H.Z.; Chen, C.R.; Li, S.J. Characterizations for optimality conditions of general robust optimization problems. J. Optim. Theory Appl. 2018, 177, 835-856. [CrossRef]

26. Lee, J.H.; Lee, G.M. On $\epsilon$-solutions for convex optimization problems with uncertainty data. Positivity 2012, 16, 509-526. [CrossRef]

27. Lee, J.H.; Jiao, L. On quasi $\epsilon$-solution for robust convex optimization problems. Optim. Lett. 2017, 11, 1609-1622. [CrossRef]

28. Sun, X.K.; Li, X.B.; Long, X.J.; Peng, Z.Y. On robust approximate optimal solutions for uncertain convex optimization and applications to multi-objective optimization. Pac. J. Optim. 2017, 13, 621-643.

29. Lee, J.H.; Lee, G.M. On $\epsilon$-solutions for robust semi-infinite optimization problems. Positivity 2018. [CrossRef]

30. Rockafellar, R.T. Convex Analysis; Princeton University Press: Princeton, NJ, USA, 1970.

31. Jeyakumar, V. Asymptotic dual conditions characterizing optimality for convex programs. J. Optim. Theory Appl. 1997, 93, 153-165. [CrossRef]

32. Loridan, P. Necessary conditions for e-optimality. Math. Program. 1982, 19, 140-152.

(C) 2018 by the authors. Licensee MDPI, Basel, Switzerland. This article is an open access article distributed under the terms and conditions of the Creative Commons Attribution (CC BY) license (http:/ / creativecommons.org/licenses/by/4.0/). 